\title{
LUIS ARAÚJO: LA VOZ TEATRAL ESPAÑOLA DE UNA CONCIENCIA HUMANITARIA GLOBAL
}

En un debate que tuvo lugar en 1993, moderado por el crítico teatral David Ladra y titulado "Conversación con el teatro alternativo", Luis Araújo (Madrid, 1956) declaró que "reconozco que el teatro es esa gran herramienta que permite mostrar en sociedad lo que la sociedad quiere ocultar" (23). Los que conocen a Luis Araújo saben que es así. Araújo está siempre dispuesto a recordarnos lo que fácil y convenientemente desatendemos. Su teatro, sus escritos sobre teatro, y su personalidad parten de un fuerte y profundo sentido de conciencia social humanitaria. Doy como ejemplo manifiesto de su actitud concienzuda lo que aparece al final de sus correos electrónicos, escrito en letras verdes: "Antes de imprimir este e-mail piense bien si es necesario hacerlo: El medioambiente es cosa de todos".

La crítica ha señalado repetidas veces que la insatisfacción con las instituciones políticas y sociales constituye el punto principal de inflexión temática en la trayectoria dramática de Luis Araújo (Ragué-Arias, 1996:236-237; Monleón, 2008). Pero la evolución de su teatro supone más que una mera desconfianza superficial de las estructuras políticas históricamente determinadas. La dramaturgia de Araújo supone una particularización en los mecanismos de dominación en las relaciones humanas en todos los planos y en distintos y variados contextos. Araújo se empeña en despertar en sus espectadores un conocimiento de su propio existir frente a los desafueros del mundo, no sólo para que se den cuenta de y llamen la atención sobre las iniquidades de que son testigos, sino para que sean conscientes de y vigilen su propio comportamiento. La relación entre un individuo y otro o entre un individuo y su entorno inmediato es de una importancia formal en las obras de Araújo. Sus obras asientan un discurso social, político y frecuentemente ontológico, motivado por un compromiso ético y moral para con la condición humana. Las sondas de dicho discurso interrogatorio del dramaturgo y el recurso artístico más eficaz de su conciencia humanitaria, son sus protagonistas, los cuales desentierran, combaten y contrarrestan los males sociales por los papeles que desempeñan.

Araújo escribe un teatro explícito y, por lo tanto, inquietante. Abarca temas como el abuso del poder, la inmigración, el SIDA, la guerra, la explotación sexual, la corrupción de las instituciones sociales y políticas, la discriminación, la apatía del público por el arte, las prácticas inhumanas de la ciencia, y la vacuidad y mezquindad humana, entre otros. Sus obras turban y desconciertan porque el autor descarta la observación y representación objetiva de la realidad, prefiriendo penetrar e indagar en la amalgama de distintas capas que se superponen para crear lo que se 
hace pasar por realidad. Araújo se empeña en examinar la sociedad en busca de sus defectos que se esconden bajo la superficie, urgiendo que sus espectadores se conciencien de las construcciones que encubren dichos defectos.

Su primera pieza, Las aventuras y andanzas del Aurelio y la Constanza (1982), es un drama infantil. Su trama es sencilla y cuenta las aventuras de Aurelio y Constanza quienes con el Capitán Mascavidrios y su ayudante Pintarroja superan una serie de obstáculos antes de encontrar el tesoro que buscan. Los personajes encarnan una doble existencia en el marco de la representación teatral. Aparecen como actores y marionetas a lo largo del drama: Aurelio-A (Aurelio Actor) y Aurelio-M (Aurelio Marioneta), Constanza-A (Constanza-Actriz) y Constanza-M (Constanza-Marioneta). El dramaturgo utiliza las marionetas en momentos climáticos o de suspenso y a los actores en escenas que dramatizan relaciones o sentimientos personales, como anota Carys Evans-Corrales (2001:17). Para entender la dualidad existencial de los personajes de Las aventuras y andanzas del Aurelio y la Constanza conviene recordar que la caracterización de todo personaje de Luis Araújo se basa en la inseparabilidad de conciencia artística y conciencia social.

Los personajes de esta pieza sostienen el contexto infantil del drama, pero también muestran cómo la complejidad formal de obras teatrales puede contribuir a crear una conciencia social. David Hawkes (2003) y Giles Gunn (2001) describen la globalización, en términos generales, como un proceso mediante el cual los asuntos regionales o locales de sociedades o culturas resuenan a nivel mundial. Mientras evoluciona la acción, Araújo funde la perspectiva infantil con la adulta para asentar su mensaje, como se demuestra en la escena en que aparece Neptuno para quejarse de la lluvia ácida, las botellas de vidrio y plástico, el vertido de petróleo y la materia radioactiva que contaminan sus aguas. Desde la perspectiva de los espectadores jóvenes es natural que el Rey del Mar se preocupe por la condición descuidada de su dominio, lo cual se podría denominar un asunto regional o local de acuerdo con las definiciones de Hawkes y Gunn. Desde la perspectiva del lector adulto, el realismo descarnado de las aguas de Neptuno recuerdan semejantes situaciones deplorables del mundo actual, lo cual se podría denominar un asunto global, de nuevo conforme a las definiciones de Hawkes y Gunn. A pesar de la perspectiva que se adopte en Las aventuras y andanzas del Aurelio y la Constanza, Araújo logra despertar la conciencia de todos para recordarles que el medioambiente es la responsabilidad de todos.

La conciencia de nuestro propio existir es esencial para crear una conciencia social individual y colectiva (Burkle, 1966; Natanson, 1952). En el teatro de Araújo un fuerte sentido del yo es el primer paso hacia el establecimiento de una responsabilidad ética frente a la sociedad. En Carmen Privatta (1988) y La parte contratante (1991), Araújo adelanta su convicción personal que el arte "es una forma de investigación sobre uno mismo, sobre la sociedad, sobre el mundo, sobre la realidad" ("Entrevista". Gabriele, 2001:217).

El personaje de Carmen Privatta es una cantante que se pasa todo el drama esperando a alguien que le acompañe con el piano. Canturrea, charla en 
voz alta con los espectadores, y bebe whiskey hasta emborracharse mientras espera que llegue su acompañante. El drama es un monólogo extendido, utilizado por Araújo para recalcar una crisis existencial. Según explica Deborah Geis, el monólogo es una estrategia a que los dramaturgos recurren frecuentemente para representar a nivel del personaje individual una crisis de envergadura mucho más universal (1993:7-27). El aislamiento emocional que siente Carmen es la base de su crisis. Mientras espera al pianista, dialoga con los espectadores a los cuales dirige sus preguntas y comunica sus ansiedades, dudas e inseguridades. Lo que más le preocupa es asegurarse de que vuelvan los espectadores. "Oye, en serio, tenéis que volver mañana", le grita al público. "¿Vais a venir? ¡Ay! Cómo podría yo convenceros..., bueno, al fin y al cabo, lo hemos pasado bien, ¿No?... ¿No? ¿Sólo me lo he pasado bien yo? [...]. En serio, de verdad que es que si no..., es que si no..." (1995:35). Para Carmen Privatta, la presencia de los espectadores en la función es esencial para llegar a una conciencia de su ser y afirmar su identidad.

La crisis de la cantante de Araújo es causada por un sentimiento de extrañeza, un sentimiento que tarde o temprano asalta a todo individuo que se enjaula dentro de sí mismo, protegiendo lo suyo y evitando todo lo que se considere el otro. Pero, también, como demuestra la situación de Carmen, todo individuo, tarde o temprano se ve obligado a confrontar la otredad para tomar conciencia de su propia individualidad por difícil que sea el enfrentamiento. En un mundo siempre más global, hay una tendencia, según Mel van Elteren, de interiorizar dentro de nosotros mismos lo que es diferente de nosotros en la sociedad, lo que no entendemos o lo que tememos; es decir, el otro. Por consiguiente, llegar a una mayor compresión de ese otro interior, lo que van Elteren llama "la interacción compleja entre el yo y la sociedad", (1999:36) es el primer paso hacia una conciencia de envergadura más universal, de una comprensión más global de la sociedad. En el escenario frente a la audiencia, Carmen se enfrenta consigo misma y con la sociedad, al mismo tiempo.

En La parte contratante, Araújo sondea la interacción operativa de lo social y lo estético que gobierna la formación de conceptos como el yo y la otredad, el pasado y el presente, el actor y el personaje, y el mito y la realidad que figuran tan prominentemente en el teatro de nuestros días (Birringer, 1991). Lo que transcurre entre los dos personajes parte de una premisa sencilla: el verdadero teatro no existe sin público. El actor ensaya y el autor escribe, pero cuestionan repetidas veces el propósito de sus respectivas acciones. Se frustran porque se dan cuenta que la relación tradicional entre autor (el productor del mensaje) y actor (el que encarna el mensaje) no da con el resultado esperado. Antes de terminar la obra, el Autor "se dispone a disparar" al Actor pero se pausa (1996:40), "se dirige al proscenio" y "entrega el fusil al público". Una voz anuncia que "faltan cinco segundos para que finalice la representación". Escribe Araújo en la acotación final: "Si algún espectador dispara el fusil, actor 
y autor caen al suelo al tiempo que se produce el oscuro..., si nadie dispara..., autor y actor" se quedan "inmóviles frente al público hablando". Al final, el Autor grita "Oscuro" (41).

Esta pieza ilustra que el teatro es una empresa tanto social como estética y subraya el doble papel del autor teatral como poder creativo y político. Araújo recalca que se trata de un texto "sobre la búsqueda de responsables..., en la sociedad y de la sociedad" (1996:28). Igual que las dos obras anteriores, en $L a$ parte contratante Araújo juega con los límites del arte teatral y, por extensión, del mundo objetivo que habita el espectador. Su mensaje resuena con el de tantos otros autores de nuestros días que ven en el teatro una herramienta eficaz para asegurar que el espectador sea co-partícipe del hecho teatral durante y después de la función. El arte teatral, como sugiere esta obra, no tiene confines. Lo que transcurre en el escenario traspasa sus límites para involucrar a los espectadores y a la sociedad, en general, para pedir y obligarles a ser activos.

Los personajes de Trenes que van al mar, del año 2001, (Clara: una crítica de arte, y Felipa: su asistenta que nunca ha visto el mar) se preparan para viajar a la playa. Durante el trayecto, las dos mujeres hablan de sus pasados y de sus deseos, y de los secretos que albergan. Confrontan aspectos de sus vidas que hasta ahora no han podido asimilar. Por consiguiente, el viaje les resulta inquietante porque se trata de viajar en busca de sus identidades. Su diálogo está contextualizado por una inflexión existencialista. La una se refleja en la otra; por cierto, se trata de otro juego del yo y del otro. Lo que cuenta Clara sirve para que Felipa progrese en la búsqueda de respuestas sobre su propia existencia, y viceversa. En Trenes que van al mar, Araújo hurga en la habilidad natural del ser humano de ocultarse a y en sí mismo, y dramatiza la difícil tarea de conocerse como se revela en el último intercambio entre las dos mujeres

Felipa: ...¿¿De qué tienes miedo?

Clara: ¿Tú nunca te has mirado en el espejo? Quiero decir ¿Durante mucho tiempo... una hora... mirándote a los ojos?

Felipa: ¿Para qué?

Clara: Para saber quién eres.

Felipa: ¿No sabe usted quién es?

Clara: ¿Tú sí?

Felipa: No sé... supongo.

Clara: ¿Quién eres?

Felipa ¿Quién soy yo?

Clara: En el espejo.

Felipa: ¿Qué espejo?

Clara: ¿Qué más da?

Felipa: Doña Clara... ¿Por qué tenemos miedo? (83-85)

(Silencio. Lentísimo oscuro mientras oímos un tren que se detiene en la estación. Sobre oscuro total, arranca el tren de nuevo). 
La pregunta, por cierto no contestada, va dirigida a los espectadores que ahora deben emprender su viaje como sugiere el tren que se arranca. Es un drama de incertidumbres epistemológicas con inferencias gnómicas. La aguda incisión en el conocimiento de sí mismo es una de las constantes de la dramaturgia de Araújo y una de las claves de su dramaturgia comprometida, conocimiento que da, eventualmente, una mayor comprensión de lo que nos rodea.

Araújo concibe el hecho teatral como imitación y reproducción de la realidad de sus espectadores y lo que encubre dicha realidad. En Vanzetti (1994), esa realidad es la situación desesperada del anarquista italiano a quien se le acusó de un crimen que no había cometido. Araújo se enfrenta con lo histórico "no para presentarlo como hecho dado y concluido", como diría Fernando de Toro, "sino para cuestionarlo, para repensarlo, para reinterpretarlo" (1994:32). Se propone cuestionar la versión oficial de lo ocurrido para hacernos conscientes de los aspectos desagradables de la sociedad. El portavoz del mensaje del autor es el propio Vanzetti, en una carta dirigida a su hermana

La cooperación ilegal entre la policía federal, la del Estado y la del distrito ha llevado nuestra causa a la jurisdicción federal, al dominio jurídico internacional. Hasta hace poco el gobernador Fuller contaba con lavarse las manos en este asunto, pero nuestras razones, nuestras pruebas, son irrefutables para la razón humana. El gobernador ha recibido peticiones de indulto de cincuenta millones de personas. Si no eres capaz de entenderlo, si papá no es capaz de entender lo que aquí se está jugando, iyo no tengo la culpa! (39).

En Vanzetti, la intención de Araújo no es historiar sino ostentar el pasado y mostrar cómo lo político y el abuso de poder emergen en forma de discurso dominante en lo que aceptamos como historia. El dramaturgo nos invita a participar en un debate ideológico y estético para crear una conciencia de la interacción compleja entre lo factual y lo ficticio en la construcción de los hechos históricos. No debemos olvidar casos como el de Vanzetti porque son referentes instructivos para el presente y el futuro. El caso de Vanzetti reverberó a nivel universal. El mensaje de Luis Araújo al respecto también repercute a nivel universal. Como en todas sus obras, Araújo se empeña en sondear lo particular con fines críticos universales.

Con una visión cada vez más negativa, escéptica y probatoria, este dramaturgo asienta en su pensamiento la convicción de que en todas las relaciones interpersonales hay trato abusivo, como se demuestra en Fantastic calentito (1985), obra en la cual examina "al género humano", como explica Jerónimo López Mozo "a través de la mirada de unos extraños mutantes" que habitan un mundo nuevo que ha surgido tras un desastre atómico" (1997:7). Grima, uno de los mutantes que nació malformado como consecuencia de un desastre es doblemente víctima: primero por su malformación y luego como espécimen de experimentos y pruebas a manos de la misma sociedad responsable de su anomalía. El mundo de Fantastic calentito es deshumanizado como en Luna negra (1984) donde la muerte de uno resulta en la 
ganancia de otro. En Enemigo (2005), cuyo tema es la guerra, la vida tampoco se aprecia y ha perdido significado como tal, y en Sin novedad (2006), una víctima de SIDA se siente incapaz de hablar de su enfermedad por miedo de que se le trate como paria social. Los personajes de estos dramas son rechazados por sus respectivas sociedades por ser diferentes. Son dramas carentes de compasión humana por una falta de amor al prójimo.

Lo que pinta el dramaturgo en estas piezas es cruel e inhumano, y es precisamente mediante lo cruel y lo inhumano que Araújo precisa desenterrar los males invisibles y atrincherados de la sociedad. Explora, al estilo de Artaud, la veta primitiva de los seres humanos por gozar de poder, de reconocimiento y de un falso sentido de superioridad a pesar de las trágicas consecuencias resultantes. Ningún personaje del repertorio de Araújo encarna dicho perfil mejor que Marcos Decántor, el protagonista de Prototipo de poniente (1982).

Decántor se abruma por inventar un aparato que inmortalice a los humanos. Se obsesiona incurablemente por el poder y por un incontenible deseo de conseguir su propia inmortalidad, situación que, con el tiempo, acaba por destruirlo a él y a las más queridas personas de su entorno. La crítica de Araújo queda bien clara. El potencial del bien social de avances científicos lleva consecuencias trágicas globales. La obsesión personal de Decántor resulta en actos trágicos e inhumanitarios. El determinismo de Decántor está matizado por una crueldad despiadada. Sus acciones hacen eco de las teorías de Artaud sobre la crueldad, para quien "en el ejercicio de la crueldad hay una especie de determinismo superior [...] No hay crueldad sin conciencia, sin una especie de aplicada conciencia. La conciencia es la que otorga al ejercicio [...] su matiz cruel, pues sobreentiende que la vida es siempre la muerte de alguien" (1978:116).

La ceguera del profesor Decántor es comparable con la obsesión por lo material de los personajes de La construcción de la catedral (1992). Dado el contexto de su situación personal, los personajes ejercen su dominación sobre los otros como mejor les convenga y a pesar de las consecuencias. Todos se imponen ilimitadamente, de alguna manera u otra, por satisfacer sus incontrolables deseos egoístas. La perspectiva historicista de esta obra es intencionadamente borrosa. En ella, la figura del rey podría ser la de cualquiera de los reyes españoles o bien cualquier jefe de gobierno en cualquier momento de la historia del mundo. Araújo se niega a fijar con precisión una época histórica específica a lo largo del drama para mostrar que el egocentrismo, la vanagloria, el engreimiento y el abuso del poder no conocen límites históricos, sociales ni geográficos. Son universales, como declara inequívocamente la figura del rey al final de la obra, cuando pide expresamente a sus conciudadanos "la unión más firme y la más estrecha solidaridad contra" las "lacras sociales", añadiendo que "no somos el único país que atraviesa [...] problemas de este orden" (134). De nuevo una situación local reverbera globalmente. 
En Mercado libre (2008), obra con la cual Araújo ganó el Premio Esperpento 2008, el dramaturgo muestra qué son capaces de hacer los humanos por dinero. Personaje B es una joven prostituta indocumentada que presta sus servicios al Personaje A, un gran abogado. En este "mercado libre" el ser humano es un artículo de mercancía, los individuos se venden y se compran, como se hace explícito al principio cuando A le pregunta a B “¿Y tú? ¿Cuánto cuestas tú?” y B contesta: "Te puedo hacer una tarifa por noche. O por día. O por fin de semana" (20). En un mundo deshumanizado como el que se representa en este texto, las relaciones humanas se establecen exclusivamente a base del maltrato del prójimo y un reparto de dos papeles: o se es víctima o se es verdugo, como se demuestra repetidas veces en intercambios como el siguiente

B: Te excita hacerme daño...

A: Me fascina tenerte a mi merced. Sentir que hago contigo lo que quiero. Sin límites, sin normas, sin tapujos... A mi total capricho (42).

El personaje A no tiene escrúpulos y no duda en aprovecharse de su situación para poder abusar. B, por su parte, muestra hasta donde está dispuesta a llegar para sacar a sus tres hijos de la cárcel. Dado su aprieto como indocumentada y su deplorable condición económica, se vende por necesidad. "Podrás hacer conmigo lo que quieras..." (70), le dice a A. El hecho es que A y B están insertos en una red relacional con su entorno que les impide sobrevivir sin aprovecharse de los otros. Así es el mercado libre: unos tienen dinero y quieren comprar, otros lo necesitan y venden, o se venden.

Mercado libre, como todo el teatro de Araújo, ilustra cómo pueden darse situaciones paradójicas de la vida y hasta dónde puede llegar la ambición humana ¿Hasta dónde puede llegar la codicia humana por poseer?, pregunta Araújo en sus obras ¿Somos todos capaces de vender nuestra dignidad? ¿Hasta dónde puede llegar el deseo humano de imponer su perspectiva personal? ¿Hasta dónde estamos dispuestos a llegar para comprometer la humanidad personal y de otros? En su teatro, Araújo desentraña una imagen del mundo en el que se debaten el individuo y sus relaciones personales; lo humano ha perdido valor y el derecho de vivir se negocia.

Por lo visto, Aráújo no da señales de desistir en el proyecto de asentar su mensaje global humanitario. En su obra más reciente, Trayectoria de la bala (2011), Premio Internacional de Dramaturgia Cultura Frontal 2010, el dramaturgo persiste en desenterrar ciertas vulnerabilidades socio-culturales como puede ser la xenofobia. Es una obra que sondea los trastornos psicológicos, sociales y emocionales que sufren los individuos que se esfuerzan por integrarse en una cultura ajena pero, también, las inquietudes de los que se esfuerzan por aceptar a dichos individuos. En Trayectoria de la bala, el papel del otro le toca a una mujer iraní inmigrante. Es una dramatización lírica y conmovedora del combate entre la inclusión y la exclusión social, el miedo a y la aceptación del otro, y la tolerancia de y las supersticiones frente a lo diferente, 
pugnas ideológicas que, según demuestra Araújo, son condiciones necesarias y previas para la esperanza de un mundo mejor.

La intención de Araújo es siempre dislocar al espectador, pasivamente sentado en su butaca, para que se inquiete y acepte el envite de verse sacudido por lo que el autor representa. No se trata de una dramaturgia cómoda, sino turbadora. Más que una dificultad intelectual de comprensión, se trata de una sensación casi física de incomodidad, de desosiego, con la situación particular del personaje como reflejo de la realidad de la condición humana, en general. El mundo de Araújo carece de comportamiento ético. Sus personajes viven y sufren coartados por injusticias que el autor quiere que sus espectadores registren.

En su artículo "Mundialización del teatro", José Monleón asevera que "frente a la mundialización de los problemas y el desconcierto actual del pensamiento político, el teatro tiene en su mano la posibilidad de colocar la experiencia humana en el centro, despojándola de la acumulación de ideologías y prejuicios" (2010:24), hecho que Araújo ilustra en sus obras. El dramaturgo focaliza su atención en las contradicciones de lo que pasa frecuentemente desapercibido a nuestro lado, para ponernos a todos en guardia contra las apariencias de la realidad y contra las infinitas maneras en que la sociedad, y los que la forman contribuyen al decrecimiento del valor humano. Araújo se preocupa por la ética del comportamiento del ser humano para el bien de la condición de vida de todos; para animarnos a la restauración de un orden social humanitario y para recordarnos, como indudablemente diría el propio dramaturgo, que "la condición humana es cosa de todos".

The College of Wooster*

Department of Spanish

Wooster, OH 44691. U.S.A.

jgabriele@wooster.edu

\section{BIBLIOGRAFÍA}

Artaud, Atonin. El teatro y su doble. Trad. Enrique Alonso y Francisco Abelenda. Barcelona: Edhasa, 1978.

Araújo, Luis. Trayectoria de la bala. Colección El Teatro de Papel 12. Madrid: Primer Acto, 2011.

------ Mercado libre. Madrid: Asociación de Autores de Teatro y Consejería de Cultura de la Comunidad de Madrid, 2008.

"Enemigo", en Diagonal No 12. Consultado: Septiembre 13 del 2006. http://www.diagonalperiodico.net/Enemigo.html?id_mot=14.

------ "Sin novedad” I, en Diagonal N 31. Consultado: Junio 5 del 2006. http://www.diagonalperiodico.net/Sin-novedad-I.html?id_mot=40.

“Sin novedad" (II), en Diagonal N 32. Consultado: Junio 17 del 2006. http://www.diagonalperiodico.net/Sin-novedad-y-II.html?id_mot=41. 
------ "Prototipo de poniente", en El juego eterno. Teatro de Luis Araújo. Ed. John P. Gabriele. Madrid: Fundamentos, 2001:135-203.

------ "La construcción de la catedral", en El Juego eterno. Teatro de Luis Araújo. Ed. John P. Gabriele. Madrid: Fundamentos, 2001:51-134.

------ Trenes que van al mar. Madrid: Asociación de Autores de Teatro y Consejería de Cultura de la Comunidad de Madrid, 2001.

------ "Entrevista con Luis Araújo", en El juego eterno. Teatro de Luis Araújo. Ed. John P. Gabriele. Madrid: Fundamentos, 2001:205-223.

------ "Las aventuras y andanzas del Aurelio y la Constanza", en Obras galardonadas. Bilbao: Centro de Documentación de Títeres de Bilbao, 1997:37-68.

------ "Luna negra", en Teatro urgente. Madrid: J. García Verdugo, 1997:35-48.

------ "Fantastic calentito", en Teatro urgente. Madrid: J. García Verdugo, 1997:13-34.

------ “A propósito de La parte contratante", en Escena N 29 (1996):28.

"La parte contratante", en Escena $\mathrm{N}^{\circ} 29$ (1996):29-41.

- Vanzetti. Madrid: Visor, 1996.

------ "Carmen Privatta", en Monólogos I. Madrid: Asociación de Autores de Teatro, 1995:13-36.

“Conversación con el teatro alternativo", en Primer Acto No 248 (1993):15-26.

Birringer, Johannes H. Theatre, Theory, Postmodernism. Bloomington: Indiana UP, 1991.

Burkle, Howard R. "Jean-Paul Sartre: Social Freedom, in Critique de la Raison Dialectique”, en Review of Metaphysics 19-4 (1966):742-757.

Evans-Corrales, Carys. "The Dramatic Use of Artifice in Luis Araújo's Las aventuras y andanzas del Aurelio y la Constanza", en Estreno $\mathrm{N}^{\circ}$ 27-2 (2001):16-22-59.

Geis, Deborah R. Postmodern Theatric(k)s: Monologue in Contemporary American Drama. Ann Arbor, U. of Michigan P, 1993.

Gunn, Giles. "Introduction: Globalizing Literary Studies", en Publications of the Modern Language Association $\mathrm{N}^{\circ} 116-1$ (2001):16-31.

Hawkes, David. Ideology. London: Routledge, 2003.

López Mozo, Jerónimo. "Invitación al teatro de Luis Araújo", en Teatro urgente. Madrid: J. García Verdugo, 1997:5-11.

Monleón, José. “Mundialización del teatro”, en Las Puertas del Drama Nº 38. (2010):23-25.

"Prólogo", en Mercado libre de Luis Araújo. Madrid: Asociación de Autores de Teatro, 2008: 9-11.

Natanson, Maurice. "Jean-Paul Sartre's Philosophy of Freedom”, en Social Research $\mathrm{N}^{\circ} 19-1 / 4$ (1952):364-380.

Ragué-Arias, María-José. El teatro de fin de milenio en España (De 1975 hasta hoy). Barcelona: Ariel, 1996.

Toro, Fernando de. "Elementos para una articulación del teatro moderno: Teatralidad, deconstrucción, postmodernidad", en Quinientos años de teatro latinoamericano. Del rito a la postmodernidad. Sergio Pereira Poza (Ed.). Santiago: Instituto Internacional de Teoría y Crítica de Teatro Latinoamericano, 1994:27-37.

Van Elteren, Mel. "The Complexities of Cultural Globalization Revisited", in The Global Village: Dead or Alive? Ed. Ray B. Browne y Marshall W. Jiswick. Bowling Green, OH: Bowling Green State UP, 1999:36-56. 